\title{
A Time Dependent Inventory Model for Exponential Demand Rate with Constant Production Where Shelf-Life of the Product Is Finite
}

\author{
Mohammad Ekramol Islam', Shirajul Islam Ukil'2, Md. Sharif Uddin² \\ ${ }^{1}$ Department of Business Administration, Northern University, Dhaka, Bangladesh \\ ${ }^{2}$ Department of Mathematics, Jahangirnagar University, Savar, Bangladesh \\ Email: shirajukil@yahoo.com
}

Received 14 December 2015; accepted 24 January 2016; published 27 January 2016

Copyright $@ 2016$ by authors and Scientific Research Publishing Inc.

This work is licensed under the Creative Commons Attribution International License (CC BY). http://creativecommons.org/licenses/by/4.0/

\section{(c) (i) Open Access}

\begin{abstract}
In this paper a time dependent inventory model is developed on the basis of constant production rate and market demands which are exponentially decreasing. It advances in quest of total average optimum cost considering those products which have finite shelf-life. The model also considers the small amount of decay. Without having any sort of backlogs, production starts. Reaching at the desired level of inventories, it stops production. After that due to demands along with the deterioration of the items it initiates its depletion and after certain periods the inventory gets zero. The decay of the products is level dependent. The objective of this paper is to find out the optimum inventory cost and optimum time cycle. The model has also been justified with proving the convex property and by giving a numerical example.
\end{abstract}

\section{Keywords}

Production Inventory, Shelf-Life, Time Dependent, Demand Class, Production Rate

\section{Introduction}

Inventory is an important ingredient of any business. It is a process and place by "proper and in time" utilization of which an enterprise can save a certain amount of production cost and the inventory cost has a vital role to reduce the production cost. Unless inventories are controlled, they are unreliable, inefficient and less cost effective. During our daily lives, generally we come across two types of materials; one is perishable items or items of 
having decay and the other one is non perishable items or items of having decay or deterioration. Most of the necessary goods like cosmetics items, radio-active substances, fashion goods, pharmaceuticals, food items etc. decrease due to its finite or limited shelf-life. Due to the limited shelf-life and market demand, the stock level or inventory continuously decreases and thereby, deterioration occurs. To get the actual inventory cost, this deterioration must be taken into consideration. On the other hand, to make the inventory cost at optimum level i.e. to get the minimum inventory cost, a suitable inventory model is required which suits to meet the actual demand in the market. In minimizing inventory cost this paper proposes a time dependent inventory model with constant production rate and exponential demand of materials with small amount of decay, whereas the existing models very often ignore the production rate, instead those consider the instantaneous replenishment rate. As the production reaches at a certain level of inventory, the production stops. Satisfying the convex property and using a numerical example, the paper could justify that the objective of formulating this model is achieved. The objective of the proposed model is to get the optimum inventory cost and optimum time cycle by introducing a time dependent inventory model with constant production rate and exponential declining demand. The paper subsequently is unfolded with literature review, assumptions, notations used in the model, development of the model, numerical illustration, sensitivity analysis, conclusion and suggestions for future work in this field.

\section{Literature Review}

Sufficient numbers of works have already been done by a large number of researchers in the area of production inventory model to build the suitable inventory models. In last few years, many researchers have studied in this field and developed inventory models to solve the real life problems. In classical inventory models, the demand rate But on ground, it must not be always correct. There may be various type of demands. Demand may be linear, quadratic, exponential, time dependent, level or stock dependent, price dependent etc. Basing on the demand pattern, the firms decide how much to produce and when to produce. Harris [1] was the pioneer to study inventory model. Whitin [2] was the first researcher to develop the inventory model for fashion goods considering its little decay in the inventory. Ghare and Schrader [3] first pointed out the effect of decay in inventory analysis and discovered the economic order quantity (EOQ) model. They explained that the consumption of the deteriorating items was a negative exponential function of time. Shiraj [4] discussed the effect of just in time manufacturing system on EOQ model. Sivazlian and Stenfel [5] determined the optimum value of time cycle by using the graphical solution of the equation to obtain the economic order quantity model. Shah and Jaiswal [6] and Dye [7] established an inventory model by considering demand as a function of selling price and three parameters of Weibull rate of deterioration. Billington [8] discussed classic economic production quantity (EPQ) model without backorders or backlogs. Pakkala and Achary [9] established a deterministic inventory model for deteriorating items with two warehouses, while the replenishment rate was finite, demand was uniform and shortage was allowed. Bhaba R. Sarker, Subbasis Mukherjee and Chidambaram V. Balan [10] explained an inventory model where demand was a composite function consisting of a constant component and a variable component proportional to inventory level in a period in which decay was exponential and inventory was positive. Teng, Chern and Yang [11] developed the model with deteriorating items and shortages assuming that the demand function was positive and fluctuating with respect to time. Skouri and Papachristos [12] discussed a continuous review inventory model considering the five costs as deterioration, holding, shortage, opportunity cost due to the lost sales and the replenishment cost due to the linearly dependency on the lot size. Chund and Wee [13] developed an integrated two stages production inventory deteriorating model for the buyer and the supplier on the basis of stock dependent selling rate considering imperfect items and just in time (JIT) multiple deliveries. Applying inventory replenishment policy Mingbao Cheng and Guoquing Wang [14] expressed the inventory model for deteriorating items with trapezoidal type demand rate, where the demand rate is a piecewise linearly functions. Hassan Shavandi and Bita Bozorgi [15] developed the location of distribution centers with inventory. Sing and Pattanak [16]-[18] developed the model for deterioration and time dependent quadratic demand under permissible delay in payment. Amutha and Chandrasekaran [19] formulated the inventory model with deterioration items, quadratic demand and time dependent holding cost. Chang and Dye [20] expressed an inventory model with deteriorating items, with time varying demand and partial backlogging. Tripathy and Mishra [21] discuss the inventory model with ordering policy for weibull deteriorating items, quadratic demand and permissible delay in payments. Sarkar et al. [22] introduced and inventory model with finite replenishment rate, trade credit policy and price discount offer. Khieng et al. [23] presented a production model for the lot-size, order level inventory system with finite production rate and the effect of decay. In our model, we have used exponential decay with 
constant production rate. Ekramol [24] [25] considered various production rate assuming the demand is constant, whereas in this model we have used constant production with varying demand. Ouyang and Cheng [26] explained the inventory model for deteriorating items with exponential declining demand and partial backlogging. In this model, we have proposed exponential declining demand along with constant production rate instead of instantaneous replenishment.

\section{Assumptions}

a. Production rate is constant at any time.

b. Production starts when inventory level is zero and it stops when inventory level is highest.

c. Inventory level is highest at $\theta=t_{1}$. Since, the production stops while inventory is highest, inventory depletes quickly due to demand and decay.

d. Demand rate exponentially decreases.

e. Deteriorating or decay rate is constant and very small.

f. Decreasing rate of demand is also constant and less than decay rate for unit inventory

g. Shortages are not allowed.

h. Lead time is zero.

\section{Development of the Model}

The model is developed on the basis of exponential market demands and constant production capacity of the organization. The model is suitable for the products which have finite shelf-life and ultimately causes the products decay. At the beginning, i.e. at time $\theta=0$, the production starts with zero inventory. In this model, the production rate $\lambda$ remains constant for entire production cycle. But the demands exponentially decrease time to time, which is shown in the Figure 1.

During time $\theta=0$ to $t_{1}$, the inventory decreases at the rate of $\lambda-Q_{0} \mathrm{e}^{-\gamma \theta}-\mu I(\theta)$, as $Q_{0} \mathrm{e}^{-\gamma \theta}$ is the market demand. Here, $\mu I(\theta)$ is the decay of $I(\theta)$ inventories at instant $\theta$. By using the above arguments we can have the following differential equations,

$$
\frac{\mathrm{d}}{\mathrm{d} \theta} I(\theta)+\mu I(\theta)=\lambda-Q_{0} \mathrm{e}^{-\gamma \theta}
$$

The general solution of the differential equation is,

$$
I(\theta)=\frac{\lambda}{\mu}-\frac{Q_{0} \mathrm{e}^{-\gamma \theta}}{\mu-\gamma}+A \mathrm{e}^{-\mu \theta}
$$

We now apply the following boundary condition, at $\theta=0$, we get, $I(\theta)=0$

By solving we get, $A=\frac{Q_{0}}{\mu-\gamma}-\frac{\lambda}{\mu}$,

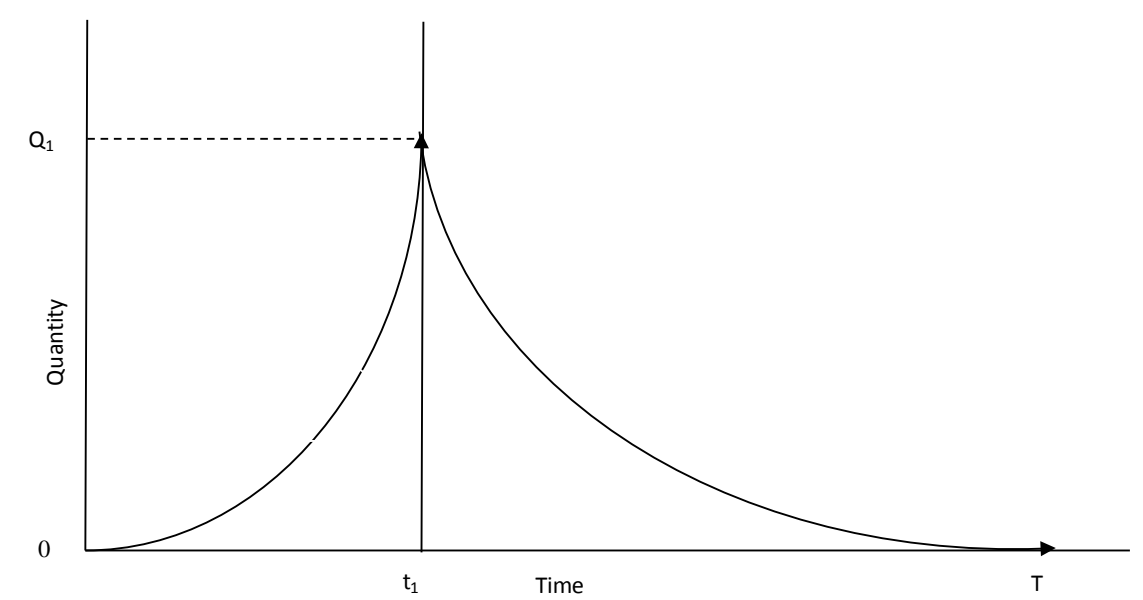

Figure 1. Time verses quantity. 
Therefore,

$$
I(\theta)=\frac{\lambda}{\mu}\left(1-\mathrm{e}^{-\mu \theta}\right)+\frac{Q_{0}}{\mu-\gamma}\left(\mathrm{e}^{-\mu \theta}-\mathrm{e}^{-\gamma \theta}\right)
$$

Applying the other boundary condition, we get $I(\theta)=Q_{1}$ at $\theta=t_{1}$. Then Equation (1) gives us,

$$
Q_{1}=\frac{\lambda}{\mu}\left(1-\mathrm{e}^{-\mu t_{1}}\right)+\frac{Q_{0}}{\mu-\gamma}\left(\mathrm{e}^{-\mu t_{1}}-\mathrm{e}^{-\gamma t_{1}}\right)
$$

Hence, the total un-decayed inventory during $\theta=0$ to $t_{1}$,

$$
\begin{aligned}
I_{1} & =\int_{0}^{t_{1}} I(\theta) \mathrm{d} \theta \\
& =\int_{0}^{t_{1}}\left[\frac{\lambda}{\mu}\left(1-\mathrm{e}^{-\mu \theta}\right)+\frac{Q_{0}}{\mu-\gamma}\left(\mathrm{e}^{-\mu \theta}-\mathrm{e}^{-\gamma \theta}\right)\right] \mathrm{d} \theta \\
& =\frac{\lambda}{\mu}\left(t_{1}+\frac{\mathrm{e}^{-\mu t_{1}}-1}{\mu}\right)-\frac{Q_{0}}{\mu-\gamma}\left(\frac{\mathrm{e}^{-\mu t_{1}}-1}{\mu}-\frac{\mathrm{e}^{-\gamma t_{1}}-1}{\gamma}\right) \\
& =\frac{\lambda}{\mu} t_{1}+\frac{\lambda}{\mu^{2}}\left(\mathrm{e}^{-\mu t_{1}}-1\right)-\frac{Q_{0}}{\mu(\mu-\gamma)}\left(\mathrm{e}^{-\mu t_{1}}-1\right)+\frac{Q_{0}}{\gamma(\mu-\gamma)}\left(\mathrm{e}^{-\gamma t_{1}}-1\right) \\
& =\frac{\lambda}{\mu} t_{1}+\frac{\lambda}{\mu^{2}}\left(-\mu t_{1}+\frac{\mu^{2} t_{1}^{2}}{2}\right)-\frac{Q_{0}}{\mu(\mu-\gamma)}\left(-\mu t_{1}+\frac{\mu^{2} t_{1}^{2}}{2}\right)+\frac{Q_{0}}{\gamma(\mu-\gamma)}\left(-\gamma t_{1}+\frac{\gamma^{2} t_{1}^{2}}{2}\right)
\end{aligned}
$$

Neglecting the higher power of $\mu$ and $\gamma$, it becomes,

$$
\begin{aligned}
& =\frac{\lambda}{\mu} t_{1}-\frac{\lambda}{\mu} t_{1}+\frac{\lambda}{2} t_{1}^{2}+\frac{Q_{0}}{\mu-\gamma} t_{1}-\frac{Q_{0} \mu}{2(\mu-\gamma)} t_{1}^{2}-\frac{Q_{0}}{\mu-\gamma} t_{1}+\frac{Q_{0} \gamma}{2(\mu-\gamma)} t_{1}^{2} \\
& =\left(\frac{\lambda}{2}-\frac{Q_{0} \mu}{2(\mu-\gamma)}+\frac{Q_{0} \gamma}{2(\mu-\gamma)}\right) t_{1}^{2}=\frac{1}{2}\left(\lambda-Q_{0}\right) t_{1}^{2}
\end{aligned}
$$

Again during $\theta=t_{1}$ to $T$, the inventory decreases at the rate of $Q_{0} \mathrm{e}^{-\gamma \theta}$. Thereby, following the process of forming first differential equation, we get the second differential equation as below:

$$
\frac{\mathrm{d}}{\mathrm{d} \theta} I(\theta)+\mu I(\theta)=-Q_{0} \mathrm{e}^{-\gamma \theta}
$$

Applying the boundary condition at $\theta=T$, we consider, $I(\theta)=0$. Then we get the general solution as below:

$$
I(\theta)=-\frac{Q_{0} \mathrm{e}^{-\gamma \theta}}{\mu-\gamma}+\frac{Q_{0} \mathrm{e}^{(\mu-\gamma) T-\mu \theta}}{\mu-\gamma}
$$

We get the following conditions, if we put the other boundary condition in equation no. (4), i.e. at $\theta=t_{1}$, $I(\theta)=Q_{1}$.

$$
Q_{1}=\frac{Q_{0}}{\mu-\gamma} \mathrm{e}^{(\mu-\gamma) T-\mu t_{1}}-\frac{Q_{0}}{\mu-\gamma} \mathrm{e}^{-\gamma t_{1}}
$$

Hence, with the help of equation no (4), we get the un-decayed inventory during $\theta=t_{1}$ to $T$, 


$$
\begin{aligned}
& I_{2}=\int_{t_{1}}^{T} I(\theta) \mathrm{d} \theta=\int_{t_{1}}^{T}\left[-\frac{Q_{0} \mathrm{e}^{-\gamma \theta}}{\mu-\gamma}+\frac{Q_{0} \mathrm{e}^{(\mu-\gamma) T-\mu \theta}}{\mu-\gamma}\right] \mathrm{d} \theta \\
&=\frac{Q_{0}}{\mu-\gamma}\left[\frac{\mathrm{e}^{-\gamma T}-\mathrm{e}^{-\gamma t_{1}}}{\gamma}\right]-\frac{Q_{0}}{\mu-\gamma}\left[\frac{\left.\mathrm{e}^{(\mu-\gamma) T-\mu T}-\mathrm{e}^{(\mu-\gamma) T-\mu t_{1}}\right]}{\mu}\right] \\
&=\frac{Q_{0}}{\gamma(\mu-\gamma)}\left(\mathrm{e}^{-\gamma T}-\mathrm{e}^{-\gamma t_{1}}\right)-\frac{Q_{0}}{\mu(\mu-\gamma)}\left(\mathrm{e}^{-\gamma T}-\mathrm{e}^{(\mu-\gamma) T-\mu t_{1}}\right) \\
&=\frac{Q_{0}}{\mu(\mu-\gamma)} e^{(\mu-\gamma) T-\mu t_{1}}+\frac{Q_{0}}{\mu \gamma} \mathrm{e}^{-\gamma T}-\frac{Q_{0}}{\gamma(\mu-\gamma)} \mathrm{e}^{-\gamma t_{1}} \\
&=\frac{Q_{0}}{\mu(\mu-\gamma)}\left[1+(\mu-\gamma) T-\mu t_{1}+\frac{1}{2}\left\{(\mu-\gamma) T-\mu t_{1}\right\}^{2}\right]+\frac{Q_{0}}{\mu \gamma}\left[1-\gamma T+\frac{1}{2} \gamma^{2} T^{2}\right] \\
&-\frac{Q_{0}}{\mu(\mu-\gamma)}\left[1-\gamma t_{1}+\frac{1}{2} \gamma^{2} t_{1}^{2}\right] \\
&= \frac{Q_{0}}{\mu(\mu-\gamma)}+\frac{Q_{0}}{\mu} T-\frac{Q_{0}}{\mu-\gamma} t_{1}+\frac{Q_{0}(\mu-\gamma)}{2 \mu} T^{2}+\frac{Q_{0} \mu}{2(\mu-\gamma)} t_{1}^{2}-Q_{0} T t_{1}+\frac{Q_{0}}{\mu \gamma} \\
&-\frac{Q_{0}}{\mu} T+\frac{Q_{0} \gamma}{2 \mu} T^{2}-\frac{Q_{0}}{\gamma(\mu-\gamma)}+\frac{Q_{0}}{\mu-\gamma} t_{1}-\frac{Q_{0} \gamma}{2(\mu-\gamma)} t_{1}^{2} \\
&=-\frac{Q_{0}}{\mu \gamma}+\frac{Q_{0} \mu}{2 \mu} T^{2}+\frac{Q_{0}}{2} t_{1}^{2}-Q_{0} T t_{1}+\frac{Q_{0}}{\mu \gamma}=\frac{1}{2} Q_{0}\left(T-t_{1}\right)^{2}
\end{aligned}
$$

And, the deteriorating items during $\theta=t_{1}$ to $T$,

$$
\begin{aligned}
D_{2} & =Q_{1}-\int_{t_{1}}^{T} R(\theta) \mathrm{d} \theta=Q_{1}-\int_{t_{1}}^{T} Q_{0} \mathrm{e}^{-\gamma \theta} \mathrm{d} \theta \\
& =\frac{Q_{0}}{\mu-\gamma} \mathrm{e}^{(\mu-\gamma) T-\mu t_{1}}-\frac{Q_{0}}{\mu-\gamma} \mathrm{e}^{-\gamma t_{1}}+\frac{Q_{0}}{\gamma}\left(\mathrm{e}^{-\gamma T}-\mathrm{e}^{-\gamma t_{1}}\right)
\end{aligned}
$$

Neglecting the higher power of $\mu$ and $\gamma$, it becomes,

$$
\begin{aligned}
= & \frac{Q_{0}}{\mu-\gamma}\left[1+(\mu-\gamma) T-\mu t_{1}+\frac{1}{2}\left\{(\mu-\gamma) T-\mu t_{1}\right\}^{2}\right]-\frac{Q_{0} \mu}{\gamma(\mu-\gamma)}\left[1-\gamma t_{1}+\frac{1}{2} \gamma^{2} t_{1}^{2}\right] \\
& +\frac{Q_{0}}{\gamma}\left[1-\gamma T+\frac{1}{2} \gamma^{2} T^{2}\right] \\
= & \frac{Q_{0}}{\mu-\gamma}+Q_{0} T-\frac{Q_{0} \mu}{\mu-\gamma} t_{1}+\frac{Q_{0} T^{2}(\mu-\gamma)}{2}+\frac{Q_{0} \mu^{2} t_{1}^{2}}{2(\mu-\gamma)}-Q_{0} \mu T t_{1}-\frac{Q_{0} \mu}{\gamma(\mu-\gamma)}+\frac{Q_{0} \mu}{\mu-\gamma} t_{1} \\
& -\frac{Q_{0} \mu \gamma t_{1}^{2}}{2(\mu-\gamma)}+\frac{Q_{0}}{\gamma}-Q_{0} T+\frac{Q_{0} \gamma T^{2}}{2} \\
= & \frac{Q_{0}}{\mu-\gamma}-\frac{Q_{0} \mu}{\gamma(\mu-\gamma)}+\frac{Q_{0}}{\gamma}+\left[\frac{Q_{0}(\mu-\gamma)}{2}+\frac{Q_{0} \gamma}{2}\right] T^{2}+\left[\frac{Q_{0} \mu^{2}}{2(\mu-\gamma)}-\frac{Q_{0} \mu \gamma}{2(\mu-\gamma)}\right] t_{1}^{2}-Q_{0} \mu T t_{1} \\
= & \frac{Q_{0} \mu}{2} T^{2}+\frac{Q_{0} \mu}{2} t_{1}^{2}-Q_{0} \mu T t_{1}=\frac{Q_{0} \mu}{2}\left(T-t_{1}\right)^{2}
\end{aligned}
$$

Total Cost Function: Total average inventory cost per unit time per cycle can be expressed as below,

$$
T C\left(t_{1}, T\right)=\frac{K+h I_{1}+\eta\left(I_{2}+D_{2}\right)}{T}
$$

By using the Equation (3), (6) and (7), we now have, 


$$
T C\left(t_{1}, T\right)=\frac{K}{T}+\frac{h}{T}\left[\frac{\lambda-Q_{0}}{2} t_{1}^{2}+\frac{Q_{0}\left(T-t_{1}\right)^{2}}{2}\right]+\frac{\eta}{T}\left[\frac{Q_{0} \mu\left(T-t_{1}\right)^{2}}{2}\right]
$$

Now, the objective is to minimize the total inventory cost $T C$. For the minimum average inventory cost $T C$ the optimum values of time $t_{1}$ and $T$ are the solutions of the following convex property:

(i) $\frac{\partial}{\partial t_{1}} T C\left(t_{1}, T\right)=0$ and $\frac{\partial}{\partial T} T C\left(t_{1}, T\right)=0$

and

(ii) $\left(\frac{\partial^{2} T C}{\partial t_{1}^{2}}\right)\left(\frac{\partial^{2} T C}{\partial T^{2}}\right)-\left(\frac{\partial^{2} T C}{\partial t_{1} \partial T}\right)^{2}>0$

The cost function will be convex if these well recognized criteria are satisfied. Thereby, we can determine the total optimum cost $T C$, optimum time interval $t_{1}^{*}$, total time cycle $T^{*}$ and the optimum order quantity $Q^{*}$. Now,

$$
\begin{aligned}
& \frac{\partial T C}{\partial t_{1}}=\frac{h}{T}\left[\left(\lambda-Q_{0}\right) t_{1}-Q_{0}\left(T-t_{1}\right)\right]-\frac{\eta}{T}\left[Q_{0} \mu\left(T-t_{1}\right)\right] \\
& \frac{\partial^{2} T C}{\partial t_{1}^{2}}=\frac{h \lambda}{T}+\frac{\eta Q_{0} \mu}{T}=\frac{1}{T}\left(h \lambda+\eta Q_{0} \mu\right) \\
& \frac{\partial^{2} T C}{\partial T \partial t_{1}}=\frac{h}{T}\left(-Q_{0}\right)-\frac{h}{T^{2}}\left(\lambda t_{1}+Q_{0}-Q_{0} T\right)-\frac{\eta}{T}\left(Q_{0} \mu\right)+\frac{\eta}{T^{2}}\left(Q_{0} \mu\right)\left(T-t_{1}\right) \\
& =\frac{-h Q_{0}}{T}-\frac{h\left(\lambda t_{1}+Q_{0}\right)}{T^{2}}+\frac{h Q_{0}}{T}-\frac{\eta Q_{0} \mu}{T}+\frac{\eta Q_{0} \mu}{T}-\frac{\eta Q_{0} \mu t_{1}}{T^{2}} \\
& =-\frac{1}{T^{2}}\left(h Q_{0}+h \lambda t_{1}+\eta Q_{0} \mu t_{1}\right)=-\frac{1}{T^{2}}\left[h Q_{0}+\left(h \lambda+\eta Q_{0} \mu\right) t_{1}\right] \\
& \frac{\partial T C}{\partial T}=-\frac{K}{T^{2}}+\frac{h}{T}\left[Q_{0}\left(T-t_{1}\right)\right]-\frac{h}{T^{2}}\left[\frac{\lambda-Q_{0}}{2} t_{1}^{2}+Q_{0} t_{1}+\frac{Q_{0}\left(T-t_{1}\right)^{2}}{2}\right] \\
& -\frac{\eta}{T^{2}}\left[\frac{Q_{0} \mu\left(T-t_{1}\right)^{2}}{2}\right]+\frac{\eta}{T}\left[h Q_{0} \mu\left(T-t_{1}\right)\right] \\
& =-\frac{K}{T^{2}}+h Q_{0}-\frac{h Q_{0} t_{1}}{T}-\frac{h\left(\lambda-Q_{0}\right) t_{1}^{2}}{2 T^{2}}-\frac{h Q_{0} t_{1}}{T^{2}}-\frac{h Q_{0}\left(T-t_{1}\right)^{2}}{2 T^{2}} \\
& -\frac{\eta Q_{0} \mu\left(T-t_{1}\right)^{2}}{2 T^{2}}+\eta Q_{0} \mu-\frac{\eta Q_{0} \mu t_{1}}{T} \\
& \frac{\partial^{2} T C}{\partial T^{2}}=\frac{2 K}{T^{3}}+\frac{h Q_{0} t_{1}}{T^{2}}+\frac{h\left(\lambda-Q_{0}\right) t_{1}^{2}}{T^{3}}+\frac{2 h Q_{0} t_{1}}{T^{3}}+\frac{h Q_{0}\left(T-t_{1}\right)^{2}}{T^{3}}-\frac{h Q_{0}\left(T-t_{1}\right)}{T^{2}} \\
& +\frac{\eta Q_{0} \mu\left(T-t_{1}\right)^{2}}{T^{3}}-\frac{\eta Q_{0} \mu\left(T-t_{1}\right)}{T^{2}}+\frac{\eta Q_{0} \mu t_{1}}{T^{2}} \\
& =\frac{2 K}{T^{3}}+\frac{h Q_{0} t_{1}}{T^{2}}+\frac{h \lambda t_{1}^{2}}{T^{3}}-\frac{h Q_{0} t_{1}^{2}}{T^{3}}+\frac{2 h Q_{0} t_{1}}{T^{3}}-\frac{h Q_{0}\left(T-t_{1}\right) t_{1}}{T^{3}}-\frac{\eta Q_{0} \mu\left(T-t_{1}\right) t_{1}}{T^{3}}+\frac{\eta Q_{0} \mu t_{1}}{T^{2}} \\
& =\frac{2 K}{T^{3}}+\frac{h \lambda t_{1}^{2}}{T^{3}}+\frac{\eta Q_{0} \mu t_{1}^{2}}{T^{3}}+\frac{2 h Q_{0} t_{1}}{T^{3}}
\end{aligned}
$$

Now, using the Equations (10), (11) and (13), we get, 


$$
\begin{aligned}
& \left(\frac{\partial^{2} T C}{\partial t_{1}^{2}}\right)\left(\frac{\partial^{2} T C}{\partial T^{2}}\right)-\left(\frac{\partial^{2} T C}{\partial t_{1} \partial T}\right)^{2} \\
& =\frac{1}{T}\left(h \lambda+\eta Q_{0} \mu\right)\left[\frac{2 K}{T^{3}}+\frac{h \lambda t_{1}^{2}}{T^{3}}+\frac{\eta Q_{0} \mu t_{1}^{2}}{T^{3}}+\frac{2 h Q_{0} t_{1}}{T^{3}}\right]-\frac{1}{T^{4}}\left(h Q_{0}+h \lambda t_{1}+\eta Q_{0} \mu t_{1}\right)^{2} \\
& =\frac{1}{T^{4}}\left[2 K h \lambda+h^{2} \lambda^{2} t_{1}^{2}+h \eta \lambda Q_{0} \mu t_{1}^{2}+2 h^{2} \lambda Q_{0} t_{1}+2 K \eta Q_{0} \mu+h \eta \lambda Q_{0} \mu t_{1}^{2}+\eta^{2} Q_{0}^{2} \mu^{2} t_{1}^{2}\right] \\
& +\frac{1}{T^{4}}\left[2 h \eta Q_{0}^{2} \mu t_{1}-h^{2} Q_{0}^{2}-h^{2} \lambda^{2} t_{1}^{2}-\eta^{2} Q_{0}^{2} \mu^{2} t_{1}^{2}-2 h^{2} \lambda Q_{0} t_{1}-2 h \eta Q_{0}^{2} \mu t_{1}-2 h \eta \lambda Q_{0} \mu t_{1}^{2}\right] \\
& =\frac{1}{T^{4}}\left[2 K h \lambda+2 K \eta Q_{0} \mu-h^{2} Q_{0}^{2}\right]
\end{aligned}
$$

This term will be greater than zero, i.e. convex property (i) will be satisfied, if $2 K h \lambda+2 K \eta Q_{0} \mu-h^{2} Q_{0}^{2}$ is greater than zero. Hence, $2 K h \lambda+2 K \eta Q_{0} \mu-h^{2} Q_{0}^{2}>0$ is to be satisfied for the minimum optimum cost. Now putting the values of (9) and (12) in the convex property (i), i.e. in $\frac{\partial}{\partial t_{1}} T C\left(t_{1}, T\right)=0$ and $\frac{\partial}{\partial T} T C\left(t_{1}, T\right)=0$ respectively and thereby we get the optimum time interval. From the equation $\frac{\partial T C}{\partial t_{1}}=0$, we get the value of $T$ as below,

$$
T=\frac{h Q_{0}+\left(h \lambda+\eta Q_{0} \mu\right) t_{1}}{Q_{0}(h+\eta \mu)}
$$

Again from $\frac{\partial T C}{\partial T}=0$, the value of $T$ can be obtained as follows,

$$
T= \pm \sqrt{\frac{\left(h \lambda+\eta Q_{0} \mu\right) t_{1}^{2}+2 h Q_{0} t_{1}+2 K}{Q_{0}(h+\eta \mu)}}
$$

Now from the equation no (9) and (10) with the positive value of $T$ from (10), we get,

$$
\frac{h Q_{0}+\left(h \lambda+\eta Q_{0} \mu\right) t_{1}}{Q_{0}(h+\eta \mu)}=\sqrt{\frac{\left(h \lambda+\eta Q_{0} \mu\right) t_{1}^{2}+2 h Q_{0} t_{1}+2 K}{Q_{0}(h+\eta \mu)}}
$$

Solving this equation we now get the value of $t_{1}$ which is mentioned here,

$$
\begin{aligned}
t_{1} & =\frac{-2 h^{2} Q_{0}\left(\lambda-Q_{0}\right) \pm \sqrt{4 h^{4} Q_{0}^{2}\left(\lambda-Q_{0}\right)^{2}-4 h Q_{0}\left(\lambda-Q_{0}\right)\left(h \lambda+\eta Q_{0} \mu\right)\left(h^{2} Q_{0}-2 h K-2 \eta K \mu\right)}}{2 h\left(\lambda-Q_{0}\right)\left(h \lambda+\eta Q_{0} \mu\right)} \\
& =\frac{-2 h^{2} Q_{0}\left(\lambda-Q_{0}\right)+\sqrt{4 h^{4} Q_{0}^{2}\left(\lambda-Q_{0}\right)^{2}+4 h Q_{0}\left(\lambda-Q_{0}\right)\left(h \lambda+\eta Q_{0} \mu\right)\left(2 \eta K \mu+h\left(2 K-h Q_{0}\right)\right)}}{2 h\left(\lambda-Q_{0}\right)\left(h \lambda+\eta Q_{0} \mu\right)}
\end{aligned}
$$

\section{Numerical Illustration}

Here, we provide a numerical illustration to justify the optimum inventory cost and the optimum order cycle. Let us consider, the inventory system has the following parameters, $K=100, h=1, \eta=0.5, \lambda=15, Q_{0}=2, \mu=0.4$, $\gamma=0.1$. With these parameters we check the condition, $2 K h \lambda+2 K \eta Q_{0} \mu-h^{2} Q_{0}^{2}=3076>0$. Now, putting all the values in equation no (5), (16), (14) and (8) we get the optimum order quantity $Q_{1}^{*}=68.62$ units, optimum order interval $t_{1}^{*}=1.418$, total time cycle $T^{*}=9.932$ units and total optimum inventory cost $T C^{*}=20.428$ units. The Figure 2 and Figure 3 show the inventory cost function verses order interval and inventory cost function verses total time cycle respectively. 


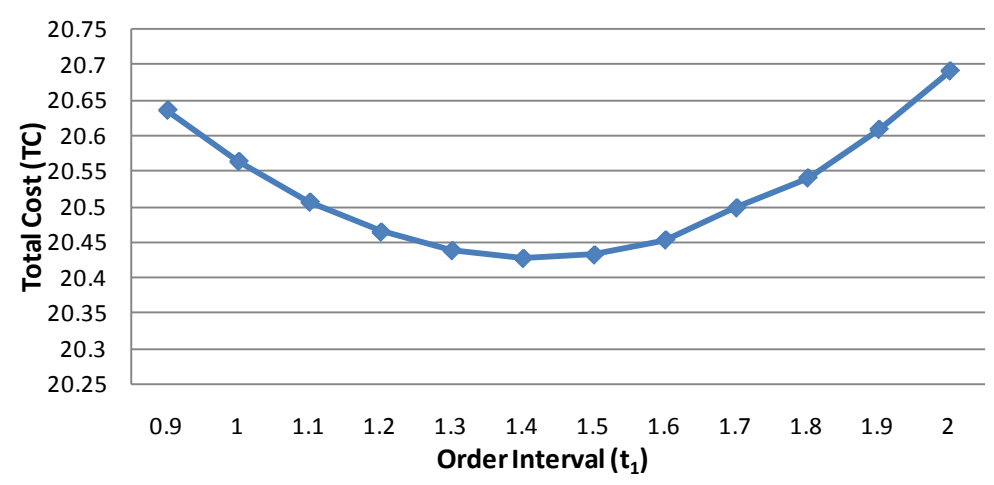

Figure 2. Order interval verses total cost.

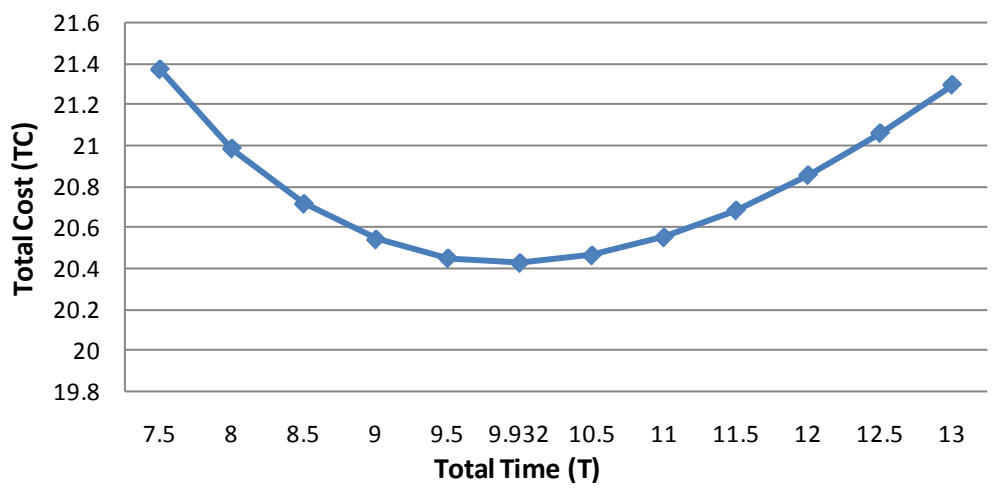

Figure 3. Total time verses total cost.

\section{Sensitivity Analysis}

Now we study the effects of changes of parameters $K, \lambda, Q_{0}, \mu, \gamma, h$ and $\eta$ on the optimal length of ordering cycle $t_{1}$, optimal time cycle $T$, optimal ordering quantity $Q_{1}$ and the minimum average total cost $T C$ per unit time in the model. We performed the sensitivity analysis by changing each of the parameters by $+50 \%,+25 \%$, $+10 \%,-10 \%,-25 \%$ and $-50 \%$ taking one parameter at a time while keeping other parameters unchanged. The details are shown in Table 1.

Analyzing the results in the above table we can summarize the following observations:

1) $t_{1}^{*}$ decreases and $T^{*}$ as well as $T C^{*}$ increase while $Q_{1}^{*}$ remain unchanged with increase in the value of the parameter $\lambda$. Here $\lambda$ is moderately sensitive to all the values.

2) $t_{1}^{*}, Q_{1}^{*}$ and $T C^{*}$ increase while $T^{*}$ decreases with increase in the value of the parameter $Q_{0}$ and $\mu$. Here $Q_{0}$ and $\mu$ are moderately sensitive to $t_{1}^{*}, T^{*}$ and $T C^{*}$ and highly sensitive to $Q_{1}^{*}$.

3) $Q_{1}^{*}$ decreases and $t_{1}^{*}, T^{*}$ and $T C^{*}$ remain unchanged with increase in the value of the parameter $\gamma$. Here $\gamma$ is moderately sensitive to $Q_{1}^{*}$.

4) $t_{1}^{*}$ decreases and $T^{*}$ as well as $T C^{*}$ increases while $Q_{1}^{*}$ remain unchanged with increase in the value of the parameter $h$. Here $h$ is moderately sensitive to all the values.

5) $t_{1}^{*}$ and $T C^{*}$ increase and $T^{*}$ decreases while $Q_{1}^{*}$ remain unchanged with increase in the value of the parameter $\eta$. Here $\eta$ is moderately sensitive to all the values are to changes.

\section{Conclusion}

In a real market, it is very unlikely that the rate of demand always remains same. At times, demand goes very high and at times it goes very low. These types of cases fit the exponential rate of demand. This proposed model expects exponential declining demand. We have developed a time dependent inventory model for the items which have finite shelf-life and time dependent demand with exponential decay. The production rate and the decay are constant all through. Total cost, order interval and total time cycle do not depend on the decreasing 
Table 1. Effects of changes in various parameters.

\begin{tabular}{|c|c|c|c|c|c|}
\hline \multirow{2}{*}{ Parameters } & \multirow{2}{*}{ Change in \% } & \multicolumn{4}{|c|}{ Value of } \\
\hline & & $t_{1}^{*}$ & $T^{*}$ & $Q^{*}$ & $T C^{*}$ \\
\hline \multirow{6}{*}{$\lambda$} & +50 & 0.923 & 14.363 & 68.620 & 21.187 \\
\hline & +25 & 1.118 & 12.148 & 68.620 & 20.808 \\
\hline & +10 & 1.280 & 10.818 & 68.620 & 20.580 \\
\hline & -10 & 1.588 & 9.046 & 68.620 & 20.276 \\
\hline & -25 & 1.937 & 7.717 & 68.620 & 20.049 \\
\hline & -50 & 3.066 & 5.501 & 68.620 & 19.669 \\
\hline \multirow{6}{*}{$Q_{0}$} & +50 & 1.766 & 6.978 & 102.930 & 24.849 \\
\hline & +25 & 1.597 & 8.160 & 85.774 & 22.638 \\
\hline & +10 & 1.491 & 9.126 & 75.481 & 21.312 \\
\hline & -10 & 1.342 & 10.917 & 61.757 & 19.544 \\
\hline & -25 & 1.222 & 12.886 & 51.465 & 18.218 \\
\hline & -50 & 0.996 & 18.794 & 34.310 & 16.007 \\
\hline \multirow{6}{*}{$\mu$} & +50 & 1.472 & 9.277 & 241.587 & 21.158 \\
\hline & +25 & 1.445 & 9.592 & 126.405 & 20.793 \\
\hline & +10 & 1.429 & 9.793 & 87.184 & 20.574 \\
\hline & -10 & 1.406 & 10.077 & 54.397 & 20.282 \\
\hline & -25 & 1.389 & 10.302 & 38.958 & 20.063 \\
\hline & -50 & 1.360 & 10.706 & 23.308 & 19.698 \\
\hline \multirow{6}{*}{$\gamma$} & +50 & 1.418 & 9.932 & 47.872 & 20.428 \\
\hline & +25 & 1.418 & 9.932 & 57.231 & 20.428 \\
\hline & +10 & 1.418 & 9.932 & 63.793 & 20.428 \\
\hline & -10 & 1.418 & 9.932 & 73.845 & 20.428 \\
\hline & -25 & 1.418 & 9.932 & 82.506 & 20.428 \\
\hline & -50 & 1.418 & 9.932 & 99.468 & 20.428 \\
\hline \multirow{6}{*}{$H$} & +50 & 1.102 & 10.433 & 68.620 & 24.878 \\
\hline & +25 & 1.234 & 10.226 & 68.620 & 22.653 \\
\hline & +10 & 1.336 & 10.063 & 68.620 & 21.318 \\
\hline & -10 & 1.514 & 9.777 & 68.620 & 19.538 \\
\hline & -25 & 1.699 & 9.484 & 68.620 & 18.203 \\
\hline & -50 & 2.208 & 8.716 & 68.620 & 15.978 \\
\hline \multirow{6}{*}{$\eta$} & +50 & 1.472 & 9.272 & 68.620 & 21.158 \\
\hline & +25 & 1.445 & 9.592 & 68.620 & 20.793 \\
\hline & +10 & 1.429 & 9.793 & 68.620 & 20.574 \\
\hline & -10 & 1.406 & 10.077 & 68.620 & 20.282 \\
\hline & -25 & 1.389 & 10.302 & 68.620 & 20.063 \\
\hline & -50 & 1.360 & 10.706 & 68.620 & 19.698 \\
\hline
\end{tabular}


rate of demand as the rate is considered very small. The model develops an algorithm to determine the optimal demand, optimal order interval, optimal time cycle and the optimum total cost. In this model, the initial demand $Q_{0}$ is highly sensitive to only optimum order quantity $Q_{1}^{*}$. All other values are moderately sensitive to changes in all the parameters. The model could be further developed considering the backlogs, varying production rate and price dependent demand rate for the perishable items. Even it could consider the non-perishable items as well.

\section{References}

[1] Harris, F.W. (1915) Operations and Costs. A. W. Shaw Company, Chicago, 48-54.

[2] Whitin, T.M. (1957) Theory of Inventory Management. Princeton University Press, Princeton, NJ, 62-72.

[3] Ghare, P.M. and Schrader, G.F. (1963) A Model for an Exponential Decaying Inventory. Journal of Industrial Engineering, 14, 238-243.

[4] Ukil, S.I., Ahmed, M.M., Sultana, S. and Uddin, Md.S. (2004) Effect on Probabilistic Continuous EOQ Review Model after Applying Third Party Logistics. Journal of Mechanics of Continua and Mathematical Science, 9, 1385-1396.

[5] Sivazlin, B.D. and Stenfel, L.E. (1975) Analysis of System in Operations Research. 203-230.

[6] Shah, Y.K. and Jaiswal, M.C. (1977) Order Level Inventory Model for a System of Constant Rate of Deterioration. Opsearch, 14, 174-184.

[7] Dye, C.Y. (1915) Joint Pricing and Ordering Policy for a Deteriorating Inventory with Partial Backlogging. Omega, 35, 184-189.

[8] Billington, P.L. (1987) The Classic Economic Production Quantity Model with Set up Cost as a Function of Capital Expenditure. Decision Series, 18, 25-42. http://dx.doi.org/10.1111/j.1540-5915.1987.tb01501.x

[9] Pakkala, T.P.M. and Achary, K.K. (1992) A Deterministic Inventory Model for Deteriorating Items with Two Warehouses and Finite Replenishment Rate. European Journal of Operational Research, 57, 71-76. http://dx.doi.org/10.1016/0377-2217(92)90306-T

[10] Sarker, B.R., Mukhaerjee, S. and Balam, C.V. (1997) An Order Level Lot Size Inventory Model with Inventory Level Dependent Demand and Deterioration. International Journal of Production Economics, 48, 227-236. http://dx.doi.org/10.1016/S0925-5273(96)00107-7

[11] Teng, J.-T., Chern, M.-S., Yang, H.-L. and Wang, Y.J. (1999) Deterministic Lot Size Inventory Models with Shortages and Deteriorating for Fluctuating Demand. Operation Research Letters, 24, 65-72. http://dx.doi.org/10.1016/S0167-6377(98)00042-X

[12] Skouri, K. and Papachristos, S. (2002) A Continuous Review Inventory Model, with Deteriorating Items, Time Varying Demand, Linear Replenishment Cost, Partially Time Varying Backlogging. Applied Mathematical Modeling, 26, 603-617. http://dx.doi.org/10.1016/S0307-904X(01)00071-3

[13] Chund, C.J. and Wee, H.M. (2008) Scheduling and Replenishment Plan for an Integrated Deteriorating Inventory Model with Stock Dependent Selling Rate. International Journal of Advanced Manufacturing Technology, 35, 665679.

[14] Cheng, M.B. and Wang, G.Q. (2009) A Note on the Inventory Model for Deteriorating Items with Trapezoidal Type Demand Rate. Computers and Industrial Engineering, 56, 1296-1300. http://dx.doi.org/10.1016/j.cie.2008.07.020

[15] Shavandi, H. and Sozorgi, B. (2012) Developing a Location Inventory Model under Fuzzy Environment. International Journal of Advanced Manufacturing Technology, 63, 191-200. http://dx.doi.org/10.1007/s00170-012-3897-6

[16] Singh, T. and Pattnayak, H. (2013) An EOQ Model for Deteriorating Items with Linear Demand, Variable Deterioration and Partial Backlogging. Journal of Service Science and Management, 6, 186-190. http://dx.doi.org/10.4236/jssm.2013.62019

[17] Singh, T. and Pattnayak, H. (2012) An EOQ Model for a Deteriorating Item with Time Dependent Exponentially Declining Demand under Permissible Delay in Payment. IOSR Journal of Mathematics, 2, 30-37. http://dx.doi.org/10.9790/5728-0223037

[18] Singh, T. and Pattnayak, H. (2013) An EOQ Model for a Deteriorating Item with Time Dependent Quadratic Demand and Variable Deterioration under Permissible Delay in Payment. Applied Mathematical Science, 7, 2939-2951.

[19] Amutha, R. and Chandrasekaran, E. (2013) An EOQ Model for Deteriorating Items with Quadratic Demand and Tie Dependent Holding Cost. International Journal of Emerging Science and Engineering, 1, 5-6.

[20] Chang, H.-J. and Dye, C.-Y. (1999) An EOQ Model for Deteriorating Items with Time Varying Demand and Partial Backlogging. Journal of the Operation Research Society, 50, 1176-1182.

http://dx.doi.org/10.1057/palgrave.jors.2600801 
[21] Tripathy, C.K. and Mishra, U. (2010) Ordering Policy for Weibull Deteriorating Items for Quadratic Demand with Permissible Delay in Payments. Applied Mathematical Science, 4, 2181-2191.

[22] Sarkar, B., Sana, S.S. and Chaudhuri, K. (2013) An Inventory Model with Finite Replenishment Rate, Trade Credit Policy and Price Discount Offer. Journal of Industrial Engineering, 2013, 18 p.

[23] Khieng, J.H., Labban, J. and Richard, J.L. (1991) An Order Level Lot Size Inventory Model for Deteriorating Items with Finite Replenishment Rate. Computers Industrial Engineering, 20, 187-197. http://dx.doi.org/10.1016/0360-8352(91)90024-Z

[24] Ekramol Islam, M. (2004) A Production Inventory Model for Deteriorating Items with Various Production Rates and Constant Demand. In: Proceedings of the Annual Conference of KMA and National Seminar on Fuzzy Mathematics and Applications, Payyanur College, Payyanur, 8-10 January 2004.

[25] Ekramol Islam, M. (2004) A Production Inventory with Three Production Rates and Constant Demands. Bangladesh Islamic University Journal, 1, 14-20.

[26] Ouyang, L.-Y., Wu, K.-S. and Cheng, M.-C. (2005) An Inventory Model for Deteriorating Items with Exponential Declining Demand and Partial Backlogging. Yugoslav Journal of Operation Research, 15, 277-288. http://dx.doi.org/10.2298/YJOR0502277O

\section{Notations}

$\lambda=$ Production rate.

$R(\theta)=Q_{0} \mathrm{e}^{-\gamma \theta}=$ The decreasing exponential demand rate at any instant $\theta$.

$\mathrm{d} \theta=$ Vary small portion of instant $\theta$.

$Q_{0}=$ Initial demand, which is any positive quantity, i.e. $Q_{0}>0$.

$\mu=$ Very small amount of constant deteriorating or decay rate for unit inventory.

$\gamma=$ Decreasing rate and $0<\gamma<\mu$.

$I(\theta)=$ Inventory level at any instant $\theta$ which is equal to $0, Q_{1}$ and 0 respectively at $\theta=0, t_{1}$ and $T$.

Here $Q_{1}$ as the highest inventory level depicts the order quantity as well.

$A, B=$ Arbitrary constants.

$I_{1}=$ Un-decayed inventory at $T=0$ to $t_{1}$.

$I_{2}=$ Un-decayed inventory at $T=t_{1}$ to $T$.

$D_{2}=$ Deteriorating inventory at $T=t_{1}$ to $T$.

$K=$ Set up or ordering cost.

$h=$ Average holding cost per unit inventory at $\theta=0$ to $t_{1}$.

$\eta=$ Average holding cost per unit inventory at $\theta=t_{1}$ to $T$.

$T C\left(t_{1}, T\right)=$ Total Cost in terms of $t_{1}$ and $T$.

$Q_{1}^{*}=$ Optimum Order Quantity.

$t_{1}^{*}=$ Optimum Order Interval.

$T^{*}=$ Total time. 